\title{
The adsorption of oxygen at GaN surfaces
}

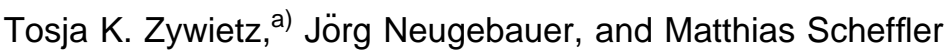 \\ Fritz-Haber-Institut der Max-Planck-Gesellschaft, Faradayweg 4-6, D-14195 Berlin, Germany
}

(Received 13 November 1998; accepted for publication 26 January 1999)

A critical point in device fabrication based on $\mathrm{GaN}$ is the controlled doping and the incorporation of impurities like, e.g., oxygen. We have explored the adsorption of oxygen at the wurtzite (0001) and $(000 \overline{1}) \mathrm{GaN}$ surfaces employing density-functional theory. Our results show that both surface orientations are very active towards oxygen adsorption, explaining the high oxygen concentrations typically observed in GaN. However, the (0001) and (0001) surfaces behave differently and oxygen incorporation is expected to be higher at the $(000 \overline{1})$ surface. The different reactivity is explained in terms of the specific structural configurations. (c) 1999 American Institute of Physics.

[S0003-6951(99)02512-7]

The control and understanding of the incorporation of dopands and in particular the unintended incorporation of impurities like, e.g., oxygen and carbon in $\mathrm{GaN}$ is still a critical issue. Experimentally, the adsorption and incorporation of oxygen at the wurtzite surfaces has been studied extensively. ${ }^{1-3}$ Theoretical $^{4}$ and experimental ${ }^{5}$ investigations have shown that oxygen is a shallow donor which is present even in nominally undoped material in rather high concentrations $\left(10^{16}-10^{19} \mathrm{~cm}^{-3}\right)$. This high concentration makes oxygen a likely candidate to cause the high background electron concentrations which are commonly observed in asgrown GaN. Recent experimental investigations demonstrated that the two polarities of the wurtzite GaN surfaces, referred to as (0001) and (0001), behave very differently: (i) The concentration of oxygen is usually higher if $\mathrm{GaN}$ is grown in the $(000 \overline{1})$ orientation. ${ }^{6}$ (ii) The (0001) surface is completely inactive against wet chemical etching with potassium hydroxide $(\mathrm{KOH})$, while the $(000 \overline{1})$ surface can be easily etched to an atomically flat surface. ${ }^{7}$ (iii) The (0001) oriented surface usually exhibits a smoother surface morphology leading to a higher material quality. ${ }^{8}$

While it has been well established that oxygen in $\mathrm{GaN}$ is a donor impurity with a very high solubility, virtually nothing is known about how oxygen is incorporated at the surface and why the two surface orientations behave so differently. In order to better understand these issues we have studied the adsorption of oxygen at the GaN (0001) and (0001) surfaces employing density-functional theory.

Total-energy calculations and geometry optimizations were performed employing density-functional theory (DFT) within the local-density approximation (LDA) and the firstprinciples pseudopotential approach. For details of the method see Ref. 9. Soft Troullier-Martins pseudopotentials ${ }^{10}$ are employed for $\mathrm{Ga}, \mathrm{N}$, and $\mathrm{O}$. The $\mathrm{Ga} 3 d$ electrons were explicitly treated as valence electrons and with a plane-wave cutoff of $60 \mathrm{Ry}$. The surfaces were modeled by supercell geometries with at least nine layers of $\mathrm{GaN}$ and a $14 \mathrm{bohr}$ vacuum region. One side of the slab was passivated by fractional pseudohydrogen. The atoms in the first two double

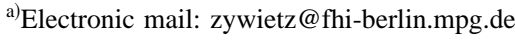

layers of the slab and the adatoms were relaxed. Relaxations below the two double layers have been found to be negligible. ${ }^{11} \mathrm{~A}(3 \times 3)$ Monkhorst-Pack mesh was used to sample the Brillouin zone. ${ }^{12}$

The energetically stable reconstructions for polar $\mathrm{GaN}$ surfaces have been recently identified for the cubic (001) and the wurtzite (0001) and (0001) orientations. ${ }^{13,14}$ A common feature found for all polar surfaces is a strong tendency towards a Ga-rich surface stoichiometry. $\mathrm{N}$ adatoms are thermodynamically unstable on almost all equilibrium surfaces. We will, therefore, focus on the two surfaces shown in Fig. 1 which are both characterized by a complete Ga surface layer. The Ga-adlayer structure [Fig. 1(b)] is one of the equilibrium surfaces observed also in experiment. ${ }^{14}$ The structure for the (0001) surface is not an equilibrium surface; a $(2 \times 2) \mathrm{Ga}$ - or $\mathrm{N}$-adatom structure is energetically more stable. ${ }^{14}$ However, as will be shown later, this surface becomes energetically preferred when oxygen is added.

Figure 3 shows the adsorption energies as a function of the oxygen coverage for both the (0001) and (0001) surfaces. ${ }^{15}$ We will now discuss the two regimes: Low oxygen coverage, which is relevant during growth, and high coverage, which is important to understand the initial oxidation step (full coverage of oxygen) of GaN surfaces, e.g., in air.

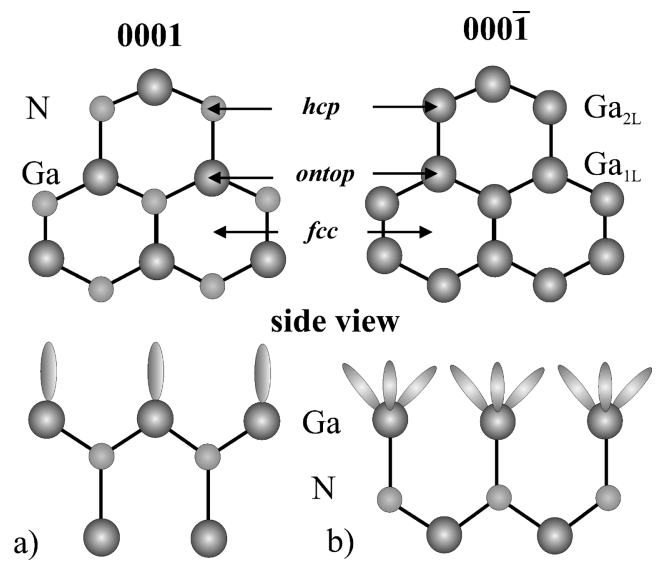

FIG. 1. Top and side view at the atomic structures of the (0001) and (0001) surfaces. 
(0001)

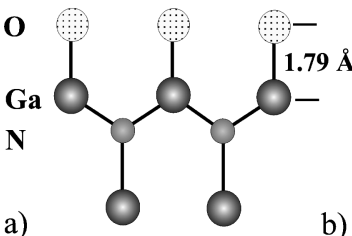

b)

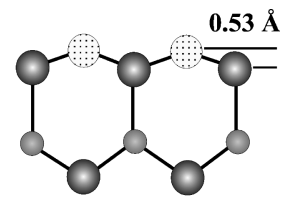

FIG. 2. Side view at the atomic structures of the (0001) and (0001) surfaces, covered with $1 \mathrm{ML}$ of oxygen.

In the low coverage regime $(\Theta \leqslant 0.25)$ we find at both orientations the fcc position to be the energetically lowest adsorption site (Fig. 2). The other sites are clearly higher in energy ( $2 \mathrm{eV}$ at the on-top position) except for the hop site at $(000 \overline{1})$, which is nearly degenerate in energy. The striking feature in Fig. 3 is the highly exothermic adsorption energy in the low coverage regime, indicating that both surfaces are highly active towards the adsorption of oxygen.

For the higher oxygen coverage, the (0001) and (0001) surfaces exhibit a strikingly different behavior: At (0001) the on-top position becomes the energetically preferred site for oxygen coverages above 0.5 monolayers (ML). The adsorption energy gets significantly less exothermic with increasing coverage and gets even endothermic above $\approx 0.8 \mathrm{ML}$. This decrease of the adsorption energy with increasing coverage reflects the strong repulsive interaction of the oxygen adatoms (see below).

For the $(000 \overline{1})$ surface, our results show a qualitatively different behavior: (i) The energetically lowest adsorption site is always the fcc position with the hcp site almost degenerate in energy and (ii) the adsorption energy gets more exothermic with increasing oxygen coverage. Thus, on the $(000 \overline{1})$ surface the interaction between oxygen adatoms is attractive.

The reason for the qualitatively different behavior at the two closed-packed surfaces can be understood in terms of the different surface structures (Fig. 2). Oxygen has a high electronegativity, and therefore, the $\mathrm{Ga}-\mathrm{O}$ bonds formed at the surface are strongly polarized towards the $\mathrm{O}$ atom making it a negatively charged ion. On the (0001) surface oxygen atoms adsorb at the on-top position (bond distance $1.79 \AA$ ).

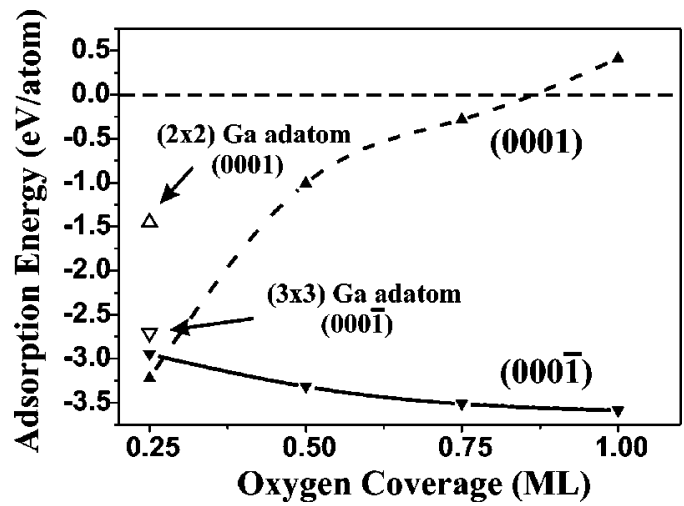

FIG. 3. Oxygen adsorption energies in eV per oxygen atom at the (0001), dashed line, and $(000 \overline{1})$; solid line, surfaces. The energy zero has been set to the energy of an isolated $\mathrm{O}_{2}$ molecule (Ref. 15). The open triangles give the adsorption energy for reconstructed surfaces with an additional Ga adatom at the hcp positions.
Consequently, there is almost no screening between the negatively charged oxygen atoms and a strong repulsive electrostatic interaction results. At $(000 \overline{1})$, on the other hand, the oxygen adatoms at the hcp (or fcc) position strongly relax into the surface layer [compare Fig. 2(b); the oxygen sits only $0.53 \AA$ above the Ga layer] resulting in an efficient electrostatic screening between the oxygen adatoms. Consequently, no repulsion and even a small attraction occurs.

From these data we can derive the following conclusions: At high oxygen concentrations (full monolayer) there is a large difference in adsorption energies of almost $4 \mathrm{eV}$ between the (0001) and $(000 \overline{1})$ surfaces, which indicates that the $(000 \overline{1})$ surface is more active towards oxygen adsorption than the (0001) surface. However, in the low coverage regime $(\theta \leqslant 0.25 \mathrm{ML})$, the adatom free $(0001)$ and $(000 \overline{1})$ surfaces behave very similarly and the different oxygen concentrations observed at (0001) and $(000 \overline{1})$ cannot be explained solely on the basis of the adsorption energies. Kinetic effects have to be taken into account.

Under growth conditions, the surface is covered with additional adatoms (like, e.g., Ga, N, or dopands), which will significantly influence the adsorption energies of oxygen. We have, therefore, also investigated the adsorption of oxygen at Ga-adatom covered surfaces $[(2 \times 2)$ and $(3 \times 3)$ reconstructions], i.e., on surfaces which contained additional Gaadatoms. While at the $(000 \overline{1})$ surface the oxygen adsorption energy changes only slightly compared to the Ga-adatom free surface, it increases by more than $1.5 \mathrm{eV}$ (per $2 \times 2$ cell) at the (0001) surface (see the triangles in Fig. 3). Therefore, the energetically most stable structure is realized when a $\mathrm{Ga}$ adatom is replaced by an oxygen atom, i.e., in the thermodynamic limit it is favorable to desorb the Ga adatom and replace it by oxygen. However, from experiment it is well established that the Ga-adatom desorption is hindered by a kinetic barrier. ${ }^{16}$ Consequently, during growth there will be a significant concentration of $\mathrm{Ga}$ adatoms at the surface. Since the presence of $\mathrm{Ga}$ adatoms significantly reduces the oxygen adsorption energy in the case of (0001), significantly higher oxygen concentrations can be stabilized on $(000 \overline{1})$.

In order to gain deeper insight into why these surfaces are so active with respect to oxygen, we analyzed the effect of oxygen on the electronic structure of the surfaces. A surface is characterized by a specific number of dangling bonds,

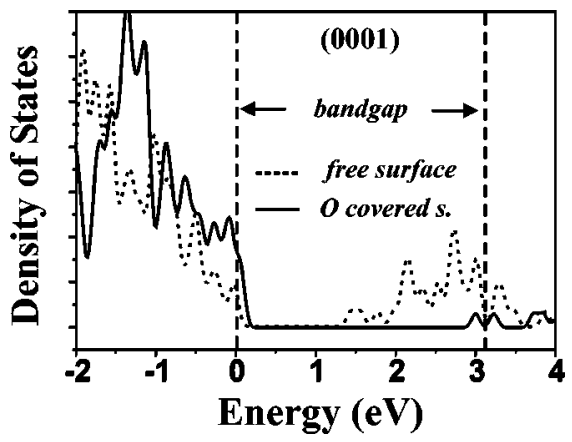

FIG. 4. Density of states (in arbitrary units) for the (0001) surface: The dotted line corresponds to the free surface, the solid line to a $1 \mathrm{ML}$ coverage of oxygen. 


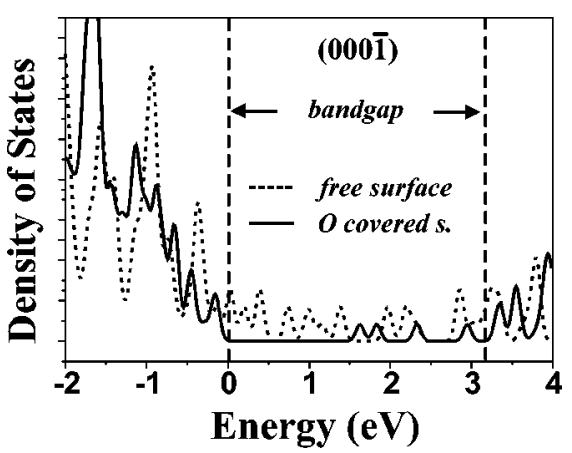

FIG. 5. Density of states (in arbitrary units) for the (0001) surface: The dotted line corresponds to the free surface, the solid line to a $1 \mathrm{ML}$ coverage of oxygen. The oxygen adsorbs at the hcp positions.

which is usually reduced by, e.g., forming dimers, trimers, or even more complicated structures in the top layer with either the cation or anion. In other words, the surfaces try to saturate the dangling bonds thereby reducing the density of states within the fundamental band gap. This mechanism is not observed at GaN surfaces, which are only cation stabilized and exhibit a metallic-like character. ${ }^{17}$ Figures 4 and 5 show the calculated density of states (DOS). Both the clean (0001) and the $(000 \overline{1})$ surfaces have a high DOS within the fundamental band gap. After adding a complete monolayer of oxygen the DOS is significantly reduced and the band gap is nearly free of surface states: The surface dangling bonds are efficiently saturated by the adsorption of oxygen, explaining the high oxygen affinity. This mechanism is not restricted to oxygen but applies to other impurities as well. Therefore, the large number of unsaturated dangling bonds makes the GaN surfaces active to a large variety of impurities explaining, e.g., why the impurity background concentrations are generally higher than in "traditional" semiconductors.

In conclusion, we have shown that the wurtzite $\mathrm{GaN}$ surfaces relevant for growth are very active against the adsorption of oxygen. In the high coverage regime, the (0001) surface generally exhibits a higher activity towards oxygen adsorption. In order to keep the oxygen incorporation as low as possible it is, therefore, preferable to grow in the (0001) orientation, which is also best to get a smooth surface morphology.

The authors gratefully acknowledge financial support from the BMBF, the "Fond der Chemischen Industrie" (T.Z.), and the "Deutsche Forschungsgemeinschaft" (J.N.).

${ }^{1}$ V. M. Bermudez, J. Appl. Phys. 80, 1190 (1996).

${ }^{2}$ K. Prabhakaran, T. G. Anderson, and K. Nozawa, Appl. Phys. Lett. 69, 3212 (1996).

${ }^{3}$ S. D. Wolter, B. P. Luther, D. L. Waltemyer, C. Önneby, S. E. Mohney, and R. J. Molnar, Appl. Phys. Lett. 70, 2156 (1997).

${ }^{4}$ J. Neugebauer and C. G. Van de Walle, Proc. ICPS 22, 2327 (1995).

${ }^{5}$ C. Wetzel, T. Suski, J. W. Ager III, E. R. Weber, E. E. Haller, S. Fischer, B. K. Meyer, R. J. Molnar, and P. Perlin, Phys. Rev. Lett. 78, 3923 (1997).

${ }^{6}$ E. S. Hellman, D. N. E. Buchanan, D. Wiesmann, and I. Brener, MRS Internet J. Nitride Semicond. Res. 1, (1996).

${ }^{7}$ M. Seelmann-Eggebert, J. L. Weyher, H. Obloh, H. Zimmermann, A. Rar, and S. Porowski, Appl. Phys. Lett. 71, 2635 (1997).

${ }^{8}$ E. J. Tarsa, B. Heying, X. H. Wu, P. Fini, S. P. DenBaars, and J. S. Speck, J. Appl. Phys. 82, 5472 (1997).

${ }^{9}$ M. Bockstedte, A. Kley, J. Neugebauer, and M. Scheffler, Comput. Phys. Commun. 107, 187 (1997); http:\www.fhi-berlin.mpg.delth\fhi96md

${ }^{10}$ N. Troullier and J. L. Martins, Phys. Rev. B 43, 1993 (1991).

${ }^{11}$ T. K. Zywietz, J. Neugebauer, and M. Scheffler (unpublished).

${ }^{12}$ H. J. Monkhorst and J. D. Pack, Phys. Rev. B 13, 5188 (1976).

${ }^{13}$ J. Neugebauer, T. K. Zywietz, M. Scheffler, J. N. Northrup, and C. G. Van de Walle, Phys. Rev. Lett. 80, 3097 (1998).

${ }^{14}$ A. R. Smith, R. M. Feenstra, D. G. Greve, J. Neugebauer, and J. E. Northrup, Phys. Rev. Lett. 79, 3934 (1997).

${ }^{15}$ The ground state of the oxygen molecule is a triplet ${ }^{3} \Sigma_{g}^{-}$state. The total energy for the $\mathrm{O}_{2}$ molecule has been calculated within the "restricted" density-functional theory. To obtain the reference energy we have, therefore, taken into account the energy difference between the singlet and triplet ground state.

${ }^{16}$ H. Yang, O. Brandt, M. Wassermeier, J. Behrend, H. P. Schnherr, and K. H. Ploog, Appl. Phys. Lett. 68, 244 (1996).

${ }^{17}$ T. K. Zywietz, J. Neugebauer, M. Scheffler, J. E. Northrup, and C. G. Van de Walle, MRS Internet J. Nitride Semicond. Res. 3, (1998). 